\author{
John J. Moskwa \& Chung-hung Pan \\ Powertrain Control Research Laboratory \\ Department of Mechanical Engineering \\ University of Wisconsin \\ Madison, WI 53706 \\ moskwa@engr.wisc.edu
}

\begin{abstract}
An important element in developing dynamic internal combustion diagnostic algorithms utilizing crankshaft speed measurements is to have a relatively accurate estimate of the load torque imposed on the engine. Without this estimate crankshaft effects from the load torque can be incorrectly attributed to cylinder pressure variations, and costly false detections can result. Cylinder pressure estimates improve considerably in accuracy when load torque estimation errors are reduced.
\end{abstract}

This paper describes the formulation of nonlinear engine load torque observers that can be used in conjunction with engine diagnostic algorithms. Simulations of these observers with and without integrators are presented to show the tracking ability during typical vehicle upshifts. Sensor noise, modeling errors, and sensor discretization effects are included in the simulations to show the robustness of these algorithms.

\section{Dynamic Transmission Model}

The nonlinear observers that are discussed in this paper are a form of model-based algorithm which can be used to estimate internal parameters or variables. As such, the dynamic model of the plant is of great importance since its structure affects the performance and accuracy or the observed variables. Because this paper focuses principally on the observer design and its performance, details of the dynamic model will not be provided herein, but the readers are urged to read [1] which specifically addressed the dynamic model in detail and its performance.

However, the authors would be remiss if they did not provide some idea of the structure of the dynamic model that is included in the nonlinear observer. A schematic diagram for the Ford AOD transmission and torque converter, as well as a clutch schedule and gear ratios are given below. This powertrain system is used in the Lincoln Town Car and the dynamic model consists of all rotating inertial components, a Ravigneaux planetary gear set, four friction clutches, two one-way clutches, two bands, and hydraulic control system. Also included is the torque converter assembly with a direct-drive lock-up clutch. This dynamic model serves as an educational tool to understand the dynamic behavior of the system, as well as an embedded model in the nonlinear observers discussed in this paper.

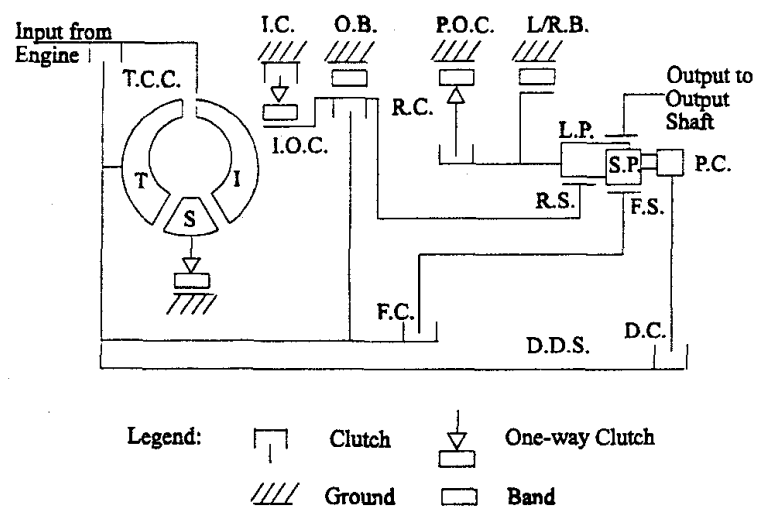

Figure - 1 Schematic Diagram of the Ford AOD system

Table 1 - Ford AOD Clutch Schedule

\begin{tabular}{|c|c|c|c|c|c|c|c|}
\hline Gear & \multicolumn{2}{|c|}{ Manual } & \multicolumn{4}{|c|}{ Over Drive Mode } & \\
\hline & $1 \mathrm{st}$ & 2nd & $1 \mathrm{st}$ & 2nd & $3 \mathrm{rd}$ & 4th & Rev. \\
\hline T.C.C & & & & & $X$ & $X$ & \\
\hline I.C. & & $\mathrm{X}$ & & $\mathrm{X}$ & $\mathrm{X}$ & $\mathrm{X}$ & \\
\hline I.O.C & & $\mathrm{X}$ & & $\mathrm{X}$ & & & \\
\hline O.B. & & $\mathrm{X}$ & & & & $\mathrm{X}$ & \\
\hline R.C. & & & & & & & $\mathrm{X}$ \\
\hline F.C. & $\mathrm{X}$ & $\mathrm{X}$ & $\mathrm{X}$ & $\mathrm{X}$ & $\mathrm{X}$ & & \\
\hline P.O.C. & $X$ & & $X$ & & & & \\
\hline L/R.B. & $\mathrm{X}$ & & & & & & $\mathrm{X}$ \\
\hline D.C. & & & & & $\mathrm{X}$ & $\mathrm{X}$ & \\
\hline $\begin{array}{l}\text { Gear } \\
\text { Ratio }\end{array}$ & 2.40 & 1.47 & 2.40 & 1.47 & 1.00 & 0.67 & 2.00 \\
\hline
\end{tabular}

The performance of this dynamic model represents typical behavior of the Ford AOD system, and time domain traces of various torques and velocities for a 1 2-3-4 upshift simulation sequence is provided below.

\section{Construction of an Engine Load Torque Observer}

Introduction

A close examination of the governing equations for the Ford AOD-E transmission system [1] shows that there are two routes to transfer combustion torque to the transmission system (Figure 1). 


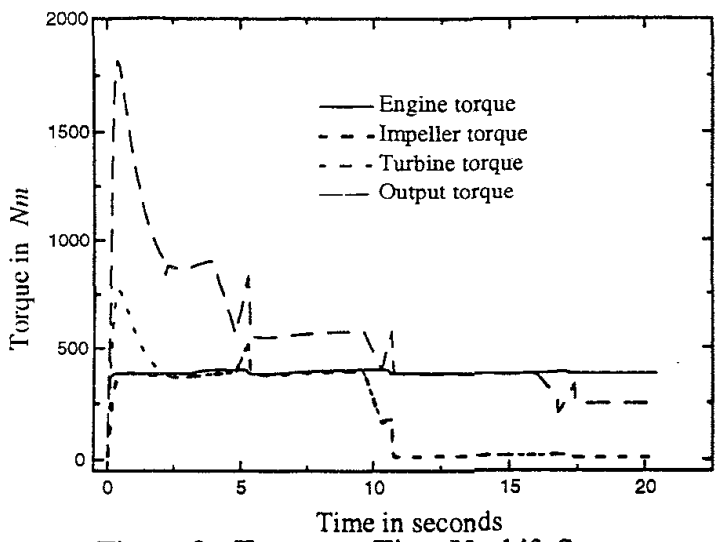

Figure 2 - Torque vs Time Upshift Sequence

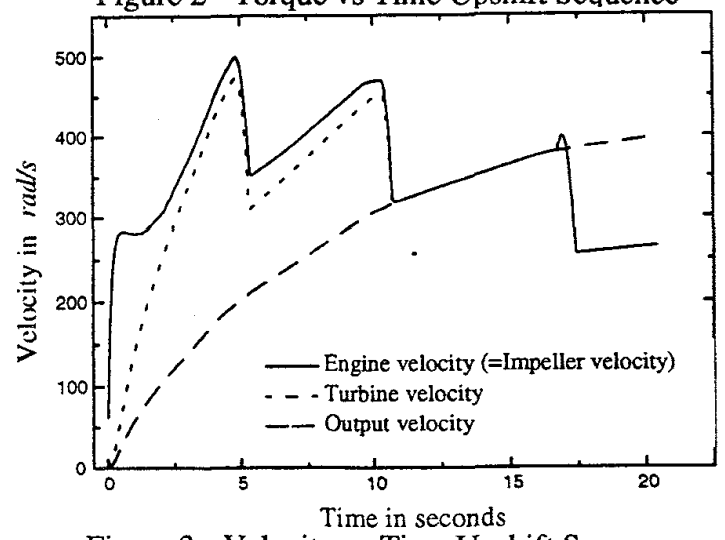

Figure 3 - Velocity vs Time Upshift Sequence

In one route, the power generated by combustion goes from the engine through the torque converter to the transmission system. The powertrain operates via this route while the vehicle is in reverse gear, first gear, 1-2 gear shifting, second gear, and 2-3 gear shifting. When the torque converter is the sole media to transfer power from the engine to the transmission system, the engine load torque could be calculated by measuring the velocity of the engine and that of the torque converter turbine. The accuracy of the engine load torque estimation relies on the perfection of the torque converter modeling and velocity measurements. For the other route, the torque converter clutch and direct drive clutch are engaged so that the combustion torque goes directly from the engine to the direct drive shaft and then into the transmission system. The powertrain operates in this route while the vehicle operates in third gear, 3-4 gear shifting and fourth gear (Table 1).

As shown in Figure 4 with the torque converter clutch and direct drive clutch engaged, the relation of the engine load torque $\left(T_{E \text { Load }}\right)$ and the direct drive shaft torque $\left(T_{P_{C}}\right)$ can be represented as Eqs. (1) and (2),

$$
\begin{gathered}
T_{E \text { Load }}=T_{E}-\left(J_{E}+J\right) \alpha_{E} \\
T_{D . C .}=T_{E . \text { load }}-J_{T} \alpha_{T}
\end{gathered}
$$

Therefore, the estimation of engine load torque can be obtained by estimating the direct drive shaft torque and measuring the turbine acceleration.

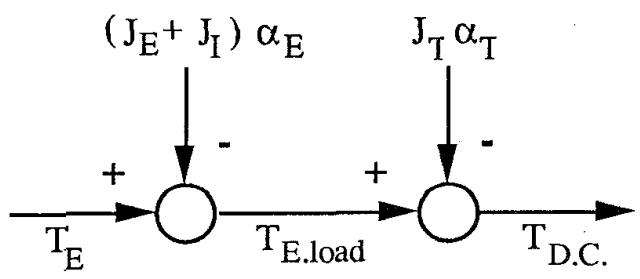

Figure 4 - Engine and Direct Drive Shaft Torque Relationships

An observer would be needed to estimate the torque going through the direct drive shaft. This research focuses on the construction of an observer to estimate the direct drive shaft torque, and the accurate estimation of the engine load torque for vehicle operations with the torque converter clutch and the direct drive clutch engaged.

\section{Observer Formulation}

The observer used in this study was adapted from the Sliding Mode observer theory. For a system with two measurable states and one observable state, the canonical form of the Sliding Mode observer is:

$$
\begin{aligned}
& \dot{\hat{\omega}}_{E}=\hat{f}_{E}\left(\omega_{E}, \omega_{P . C .} \hat{T}_{D . C .}, t\right) \\
&-h_{1} \omega_{E}-h_{2} \hat{\omega}_{P . C .}-k_{I} \operatorname{sgn}\left(s_{E}\right) \\
& \dot{\hat{\omega}}_{P . C .}=\hat{f}_{P . C .}\left(\omega_{E}, \omega_{P . C .} \hat{T}_{D . C .}{ }^{t}\right) \\
&-h_{3} \hat{\omega}_{E}-h_{4} \omega_{P . C .}-k_{2} \operatorname{sgn}\left(s_{P . C .}\right) \\
& \dot{\hat{T}}_{D . C .}=\hat{f}_{D . C .}\left(\omega_{E}, \omega_{P . C .} \hat{T}_{D . C .}, t\right) \\
&-h_{5} \omega_{E}-h_{6} \omega_{P . C .}-k_{3} \operatorname{sgn}\left(s_{E}\right)-k_{4} \operatorname{sgn}\left(s_{P . C .}\right)
\end{aligned}
$$

where the symbols with caret indicate the estimated value, the ones with tilde represent the difference between the measured values and the estimated values.

The performance of this Sliding Mode observer heavily depend on the selection of the linear gains $\left(h_{i}\right)$ and the switching gains $\left(k_{j}\right)$. Researchers have proposed several algorithms to select these gains, e.g., Constant Gain Kalman Filter, Extended Kalman Filter, Pole-placement Method and Frequency Shaping Method [2]. If the statistical characteristics of the measurement noise are known, Constant Gain Kalman Filter or Extended Kalman Filter method could serve as a guide to help users to select appropriate linear gains for the system. Misawa[3] has proposed two methods to select both linear gains and switching gains simultaneously. However, both of his methods have the problem that the system will have more variables than available equations, which forces users to add additional assumptions to carry out the algorithm. Another popular algorithm is the pole-placement method. Literature abounds with several modified versions of the poleplacement method [5-10]. Each algorithm has its own merits and limitations. A combination of the method proposed by Misawa [3] and the pole-placement method was chosen in this study. The procedure for using this algorithm is detailed in the following section.

\section{Selection of the Linear and Switching Gains}


The performance of the observer is mainly determined by the selection of the linear gains and switching gains. To secure stability and robustness, certain criteria for the selection must be met (Misawa, 1988). Compromise for fast response to change and shorter transient period must be made. An algorithm that allows users to design a stable, robust, fast-responding observer with a reasonable transient period is discussed in the following paragraphs.

As the canonical observer structure (Eqs. (3-5)) shows, there are six linear gains and four switching gains in this observer. First, the linear gains are determined by the desired response rate of the system using the poleplacement method. Then, the relationships among the switching gains are examined by the upper bound of modeling errors. Finally, the switching gains are obtained using Misawa's (1988) method. In the following section, both linear gains and switching gains are examined by the criteria proposed by Misawa (1988) to guarantee global stability and robustness.

The linear gains $\left(h_{i}\right)$ are designed by placing the poles at $\lambda_{1}, \lambda_{2}$, and $\lambda_{3}$ for the observer dynamic system matrix:

$$
\begin{aligned}
& A_{D . C .}= {\left[\begin{array}{ccc}
\frac{\partial f_{E}}{\partial \omega_{E}}-h_{1} & \frac{\partial f_{E}}{\partial \omega_{P . C .}}-h_{2} & \frac{\partial f_{E}}{\partial T_{D . C .}} \\
\frac{\partial f_{P . C .}}{\partial \omega_{E}}-h_{3} & \frac{\partial f_{P . C .}}{\partial \omega_{P . C .}}-h_{4} & \frac{\partial f_{P . C .}}{\partial T_{D . C .}} \\
\frac{\partial f_{D . C .}}{\partial \omega_{E}} h_{5} & \frac{\partial f_{D . C .}}{\partial \omega_{P . C .}}-h_{6} & \frac{\partial f_{D . C .}}{\partial T_{D . C .}}
\end{array}\right] } \\
& \therefore \operatorname{det}\left(s I-A_{D . C}\right)=\sum_{i=1}^{3}\left(s-\lambda_{i}\right)
\end{aligned}
$$

and set

$$
\begin{aligned}
& h_{2}=\kappa h_{1} \\
& h_{3}=\beta h_{4} \\
& h_{5}=\gamma h_{6}
\end{aligned}
$$

where $\kappa, \beta, \gamma$ are constants. The values of $\kappa, \beta, \gamma$ could be decided by the characteristics of the system. Eqs.

(11)-(13) are obtained by expanding Eq. (7):

$$
\begin{aligned}
& h_{1}+h_{4}-\frac{\partial f_{E}}{\partial \omega_{E}}-\frac{\partial f_{P . C .}}{\partial \omega_{P . C .}}-\frac{\partial f_{D . C .}}{\partial T_{D . C .}}=\lambda_{1}-\lambda_{2}-\lambda_{3} \text {, } \\
& \left(h_{I}-\frac{\partial f_{E}}{\partial \omega_{E}}\right)\left(h_{4}-\frac{\partial f_{P . C .}}{\partial \omega_{P . C .}}\right) \frac{\partial f_{D . C .}}{\partial T_{D . C .}}\left(h_{I}-\frac{\partial f_{E}}{\partial \omega_{E}}\right) \\
& -\frac{\partial f_{D . C .}}{\partial T_{D . C .}}\left(h_{4}-\frac{\partial f_{P . C .}}{\partial \omega_{P . C .}}\right)+\frac{\partial f_{E}}{\partial T_{D . C .}}\left(h_{5}-\frac{\partial f_{D . C .}}{\partial \omega_{E}}\right) \\
& +\frac{\partial f_{E}}{\partial T_{D . C .}}\left(h_{6}-\frac{\partial f_{D . C .}}{\partial \omega_{P . C .}}\right)-\left(h_{2}-\frac{\partial f_{E}}{\partial \omega_{P . C .}}\right)\left(h_{3}-\frac{\partial f_{P . C .}}{\partial \omega_{E}}\right) \\
& =\lambda_{1} \lambda_{2}+\lambda_{2} \lambda_{3}+\lambda_{3} \lambda_{1} \\
& -\frac{\partial f_{D . C .}}{\partial T_{D . C .}}\left(h_{1}-\frac{\partial f_{E}}{\partial \omega_{E}}\right)\left(h_{4}-\frac{\partial f_{P . C .}}{\partial \omega_{P . C .}}\right)
\end{aligned}
$$

$$
\begin{aligned}
& -\frac{\partial f_{E}}{\partial T_{D . C .}}\left(h_{3}-\frac{\partial f_{P . C .}}{\partial \omega_{E}}\right)\left(h_{6}-\frac{\partial f_{D . C .}}{\partial \omega_{P . C .}}\right) \\
& -\frac{\partial f_{P . C .}}{\partial T_{D . C .}}\left(h_{2}-\frac{\partial f_{E}}{\partial \omega_{P . C .}}\right)\left(h_{5}-\frac{\partial f_{D . C .}}{\partial \omega_{E}}\right) \\
& -\frac{\partial f_{E}}{\partial T_{D . C .}}\left(h_{4}-\frac{\partial f_{P . C .}}{\partial \omega_{P . C .}}\right)\left(h_{5}-\frac{\partial f_{D . C .}}{\partial \omega_{E}}\right) \\
& -\frac{\partial f_{D . C .}}{\partial T_{D . C}}\left(h_{2}-\frac{\partial f_{E}}{\partial \omega_{P . C .}}\right)\left(h_{3}-\frac{\partial f_{P . C .}}{\partial \omega_{E}}\right) \\
& -\frac{\partial f_{P . C .}}{\partial T_{D . C .}}\left(h_{1}-\frac{\partial f_{E}}{\partial \omega_{E}}\right)\left(h_{6}-\frac{\partial f_{D . C .}}{\partial \omega_{P . C .}}\right)=\lambda_{1} \lambda_{2} \lambda_{3} \\
& \text { Error dynamics of the observer are } \\
& \dot{\bar{\omega}}_{E}=\Delta f_{E}-h_{1} \tilde{\omega}_{E}-h_{2} \tilde{\omega}_{P . C .}-k_{1} \operatorname{sgn}\left(\tilde{\omega}_{E}\right) \\
& \dot{\hat{\omega}}_{P . C .}=\Delta f_{P . C .}-h_{3} \omega_{E}-h_{4} \omega_{P . C .}-k_{2} \operatorname{sgn}\left(\omega_{P . C .}\right) \\
& \dot{\tilde{T}}_{D . C .}=\Delta f_{D . C .}-h_{5} \omega_{E}-h_{6} \omega_{P . C .} \\
& -k_{3} \operatorname{sgn}\left(\tilde{\omega}_{E}\right)-k_{4} \operatorname{sgn}\left(\tilde{\omega}_{P . C}\right)
\end{aligned}
$$

where $\Delta f_{E}, \Delta f_{P . C}$ and $\Delta f_{D . C}$ are modeling errors for the engine acceleration, planetary carrier acceleration of the transmission system and the direct drive shaft torque changing respects to time, respectively.

The sliding surface is defined as $s=\hat{v}-v$, where $\hat{0}$ is the estimated value, $v$ is the measured value. The sliding condition, ss $<0$, of the engine velocity $\left(\omega_{E}\right)$, the planetary carrier velocity $\left(\omega_{P . C}\right)$ of the transmission system and the direct drive shaft torque are

$$
\begin{gathered}
\omega_{E}\left(\Delta f_{E}-h_{1} \tilde{\omega}_{E}-h_{2} \tilde{\omega}_{P . C .}-k_{I} \operatorname{sgn}\left(\tilde{\omega}_{E}\right)\right)<0 \\
\tilde{\omega}_{P . C .}\left(\Delta f_{P . C .}-h_{3} \tilde{\omega}_{E}-h_{4} \omega_{P . C .}\right. \\
\left.-k_{2} \operatorname{sgn}\left(\omega_{P . C .}\right)\right)<0 \\
\tilde{T}_{D . C .}\left(\Delta_{D . C .}-h_{5} \omega_{E}-h_{6} \tilde{\omega}_{P . C .}\right. \\
-k_{3} \operatorname{sgn}\left(\tilde{\omega}_{E}\right)-k_{4} \operatorname{sgn}\left(\tilde{\omega}_{P . C .}\right)<0
\end{gathered}
$$

Without the presence of measurement noise, both $\omega_{E}, \omega_{P . C \text {. }}$ are related to the estimated $T_{D . C}$ (shown in [1]). Hence, it is necessary to discuss the effect of the estimation of $T_{D . C}$ on the selection of linear gains and switching gains. If $T_{D . C}$ is over-estimated (which means the value of estimated $T_{D C}$ is larger than the real one), $\hat{\omega}_{E}$ would be smaller than $\omega_{E}$ and $\hat{\omega}_{P . C}$. would be lager than $\omega_{P . C}$. Therefore, an over-estimated $T_{D . C}$. (which means $\tilde{T}_{D_{C} .}>0$ ) results in a negative $\omega_{E}$ and a

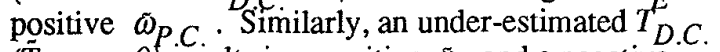
$\left(\tilde{T}_{D . C .}<0\right)$ results in a positive $\tilde{\omega}_{E}$ and a negative $\omega_{P . C .}$

These various cases can be combined into the following three equations.

$$
\begin{aligned}
& \left|\Delta f_{E}-h_{1} \tilde{\omega}_{E}-h_{2} \tilde{\omega}_{P . C .}\right| \\
\leq & \left|\Delta f_{E}\right|+\left|h_{1} \tilde{\omega}_{E}\right|+\left|h_{2} \tilde{\omega}_{P . C .}\right| \leq k_{1}
\end{aligned}
$$




$$
\begin{aligned}
& \left|\Delta f_{P . C .}-h_{3} \omega_{E}-h_{4} \omega_{P . C .}\right| \\
\leq & \left|\Delta f_{P . C .}\right|+\left|h_{3} \omega_{E}\right|+\left|h_{4} \omega_{P . C .}\right| \leq k_{2} \\
& \left|\Delta f_{D . C .}-h_{5} \tilde{\omega}_{E}-h_{6} \omega_{P . C .}\right| \\
\leq & \left|\Delta f_{D . C .}\right|+\left|h_{5} \tilde{\omega}_{E}\right|+\left|h_{6} \tilde{\omega}_{P . C .}\right| \leq k_{4}-k_{3}
\end{aligned}
$$

By solving these equations (Eqs. (11)-(13)) for $h_{1}, h_{4}, h_{6}, h_{2}, h_{3} . h_{5}$ can also be obtained (Eqs. (8)(10)). The analysis above and Eqs. (3)-(5) imply that $h_{2}, h_{3} . h_{5}$ and $k_{3}$ should be kept small or even negative.

After the calculation of the linear gains and the examination of the relationship of the switching gains, the method and criteria proposed by Misawa [3] are used to choose the switching gains and to guarantee the stability and robustness of the Sliding Mode observer. To broaden the range of application and avoid the oscillations brought by the sudden gain change [11-13], a less restrictive condition is used in this study by replacing the pure switching (signum function) to a saturation function. The saturation function is defined as:

$$
\operatorname{sgn}\left(\tilde{y}_{i}\right) \rightarrow \operatorname{sgn}\left(\tilde{y}_{i}\right)= \begin{cases}\tilde{y}_{i} / / \tilde{y}_{i} / & \text { if } / \tilde{y}_{i} / \geq \varepsilon_{i} \\ \tilde{y}_{i} / \varepsilon_{i} & \text { if } / \tilde{y}_{i} /<\varepsilon_{i}\end{cases}
$$

For details of the procedure used in selecting the switching gains, see reference [14].

For the observer of this study, matrices $A, C, D, H, K$, are defined as:

$$
\begin{gathered}
A=\left[\begin{array}{ccc}
\frac{\partial f_{E}}{\partial \omega_{E}} & \frac{\partial f_{E}}{\partial \omega_{P . C .}} & \frac{\partial f_{E}}{\partial T_{D . C .}} \\
\frac{\partial f_{P . C .}}{\partial \omega_{E}} & \frac{\partial f_{P . C .}}{\partial \omega_{r}} & \frac{\partial f_{P . C .}}{\partial T_{D . C .}} \\
\frac{\partial f_{D . C .}}{\partial \omega_{E}} & \frac{\partial f_{D . C .}}{\partial \omega_{P . C}} & \frac{\partial f_{D . C .}}{\partial T_{D . C .}}
\end{array}\right] \\
C=\left[\begin{array}{lll}
1 & 0 & 0 \\
0 & 1 & 0
\end{array}\right] \\
\rho=\operatorname{diag}(1, I) \\
\Delta=\operatorname{diag}\left(\varepsilon_{1}, \varepsilon_{2}\right) \\
\boldsymbol{K}=\boldsymbol{D} \rho=\left[\begin{array}{ll}
k_{1} & 0 \\
0 & k_{2} \\
k_{3} & k_{4}
\end{array}\right] \\
\boldsymbol{H}=\left[\begin{array}{ll}
h_{1} & h_{2} \\
h_{3} & h_{4} \\
h_{5} & h_{6}
\end{array}\right] \\
\boldsymbol{M}=\left[\begin{array}{ll}
\frac{1}{\varepsilon_{1}} & 0 \\
0 & \frac{1}{\varepsilon_{2}}
\end{array}\right] \\
\boldsymbol{A}-\left(\boldsymbol{H}+K \Delta^{-1}\right) \boldsymbol{C}=
\end{gathered}
$$

$$
\left[\begin{array}{ccc}
\frac{\partial f_{E}}{\partial \omega_{E}}-h_{1}-\frac{k_{1}}{\varepsilon_{1}} & \frac{\partial f_{E}}{\partial \omega_{P . C .}}-h_{2} & \frac{\partial f_{E}}{\partial T_{D . C}} \\
\frac{\partial f_{P . C .}}{\partial \omega_{E}}-h_{3} & \frac{\partial f_{P . C .}}{\partial \omega_{r}}-h_{4}-\frac{k_{2}}{\varepsilon_{2}} & \frac{\partial f_{P . C .}}{\partial T_{D . C .}} \\
\frac{\partial f_{D . C}}{\partial \omega_{E}}-h_{5}-\frac{k_{3}}{\varepsilon_{I}} & \frac{\partial f_{D . C .}}{\partial \omega_{P . C .}}-h_{6}-\frac{k_{4}}{\varepsilon_{2}} & \frac{\partial f_{D . C .}}{\partial T_{D . C}}
\end{array}\right]
$$

Two merits of this algorithm are that users are allowed direct control of the system response by using the poleplacement method to choose the linear gains matrix and users do not need to add more assumptions to implement Misawa's [3] method. The usage of Misawa's [3] criteria guarantees the stability and robustness of the observer.

\section{Sliding Mode Observer with Integrators}

The other observer tested in this study incorporates the use of integrators in the Sliding Mode observer in order to improve tracking. Similar to the benefit of controllers with integrators, the addition of integrators will improve the steady state errors by one order. It is also shown in the literature that adding integrators will allow systems to have smaller gains and provide better tracking. The use of smaller gains will minimize noise amplification and the improved tracking will increase the system performance.

For the Sliding Mode observer with integrators, the sliding surfaces for engine velocity, and planetary carrier velocity are defined as:

$$
\begin{gathered}
\dot{s}_{E}=\dot{\tilde{\omega}}_{E}+\xi_{1} \tilde{\omega}_{E}=-k_{1}^{\prime} \operatorname{sgn}\left(s_{E}\right) \\
\dot{s}_{P . C .}=\dot{\tilde{\omega}}_{P . C .}+\xi_{1} \tilde{\omega}_{P . C .}=-k_{2}^{\prime} \operatorname{sgn}\left(s_{P . C .}\right)
\end{gathered}
$$

and the observer has similar forms and restrictions as the Sliding Mode observer without integrator (Eqs. (3)-(5)).

\section{Simulations and Results}

\section{Introduction}

Two observer designs, the Sliding Mode observer with and without integrators, are investigated. A sensitivity study was undertaken to explore the robustness of these observers to measurement and modeling errors, linear and feedback gains, and other effects that could be reasonable expected to occur. However, due to space constraints a limited number of simulations are presented in this paper. The reader is encouraged to read [14] for details of these studies. Four velocities are measured in this study: the engine velocity, the turbine velocity of the torque converter, the planetary carrier velocity and the ring gear velocity of the transmission system. Most vehicles today have already installed sensors to measure these velocities [15]. Therefore, few or no additional modifications are required to be made on the transmission in order to implement the observers on many modern transmission systems. The engine velocity and the turbine velocity of the torque converter are measured to calculate the impeller torque and the 
turbine torque [1]. The engine velocity and the planetary carrier velocity of the transmission system are used as two measurable states for the observers. For the inertia phase of 3-4 gear shifting, which will be examines here, the ring gear velocity of the transmission system is needed to estimate the engine load torque.

The velocity data used in this study are obtained in the following procedure. First, the velocity data are calculated from the powertrain model, which represents the exact velocity. Second, a Gaussian random noise function with an intensity of $100 \mathrm{rpm}$ is added to the data to simulate the high frequency noise. Third, based on the corrupted data, the measured data are calculated using the discrete sensor model, such as a toothed wheel or discrete optical signal (the Ford AOD system has a 164 tooth flywheel). Finally, the data used in the observers are obtained by applying the Fast Fourier Transform (FFT) method to filter out high frequency noise and reduce the measurement errors caused by sensors. By this method a realistic assessment of the observer's capabilities can be made.

Two of the governing equations for all transmission operations considered (i.e.: third gear, 3-4 shifting, and fourth gear) are presented as Eqs. (36)-(37).

$$
\begin{gathered}
\dot{\hat{\omega}}_{E}=\frac{T_{E}-T_{I}-T_{D . C .}}{J_{E}} \\
\hat{T}_{D . C .}=G\left(\omega_{E}-\omega_{P . C .}\right)
\end{gathered}
$$

The other equations for the observer design under different gear operations are:

For third gear,

$\dot{\omega}_{P . C .}=\frac{T_{D . C .}+T_{T}-T_{r}}{J_{T . S .}}$

For the torque phase of 3-4 gear up-shifting,

$\dot{\hat{\omega}}_{P . C .}=\frac{T_{D . C .}+T_{T}-T_{r}-T_{O . B} .}{J_{T S .}}$

For the inertia phase of 3-4 gear up-shifting,

$\dot{\omega}_{P . C .}=\frac{T_{D . C .}+T_{T}-T_{r}-T_{O . B}}{J_{T . S .}}-J \dot{\omega}_{r}$

For fourth gear,

$\dot{\hat{\omega}}_{P . C .}=\left(\frac{n_{r . s .}+n_{r}}{n_{r}}\right) \frac{\left[T_{D . C .}-\left(\frac{n_{r . s .}+n_{r}}{n_{r}}\right) T_{r}\right]}{J_{T . S .}}$

where $J_{\text {T.S. }}=J_{\text {f.s. }}+J_{\text {r.s. }}+J_{P . C .}+J_{r}+J_{\text {l.p. }}+J_{s . p .}$.

The comparisons of the performances of the Sliding Mode observers with and without integrators in 3-4 gear shifting are presented in Figure 5. The data used in these simulations are obtained following the procedure outlined earlier in this section.
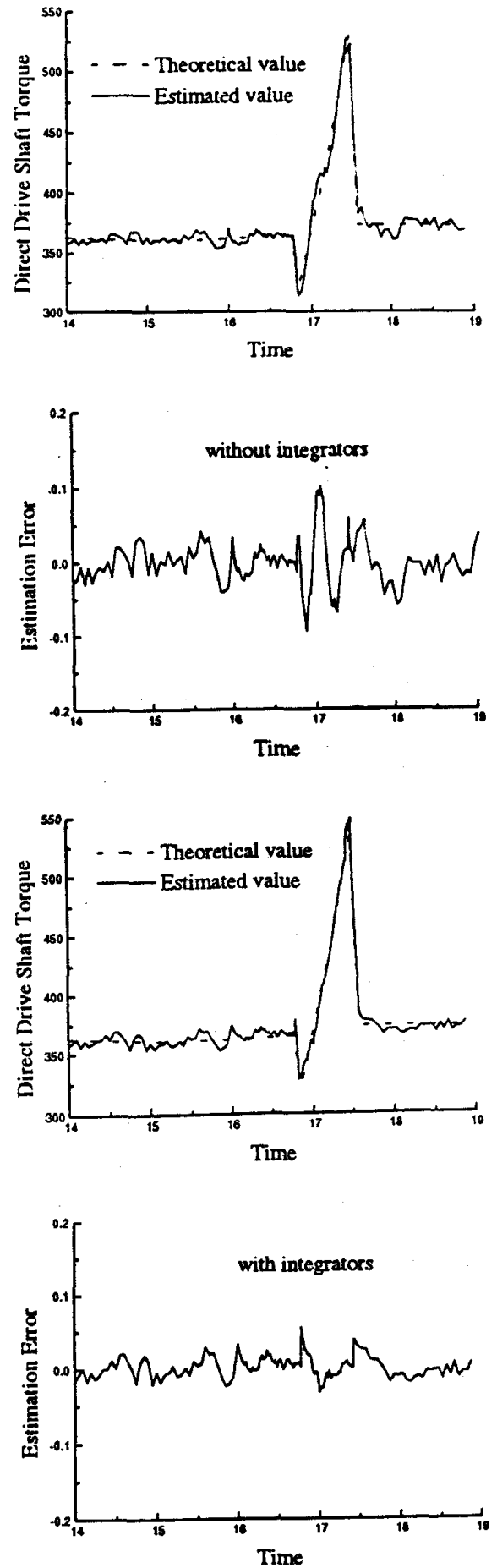

Figure 5 - Simulation Results of Sliding Mode Observers with and without Integrators

As shown in Figure 5, the tracking in the transient states have been improved by the use of integrators because of the enhanced tracking ability. The comparisons of Figure 5 indicate that the steady state errors of the 
Sliding Mode observer with integrators are smaller than those of Sliding Mode observer without integrators. The decrease of the switching gains would usually result in longer transient periods. However, with the integrators, even though the switching gains matrix used in the Sliding Mode observer with integrators is smaller than the one used in the Sliding Mode observer alone, the transient periods remain about the same. It is shown that, with the integrators, the observer is allowed to have smaller gains without prolonging the transient periods, and is able to have better tracking as well as smaller steady state errors. Since the Sliding Mode observer with integrators has better performance than the one without integrator for this application, the following simulation results are mainly based on the Sliding Mode observer with integrators.

Since the estimation of the engine load torque is obtained by estimating the direct drive shaft torque and measuring the turbine acceleration, the estimation error of the engine load torque would be affected by both direct drive torque estimation error and turbine acceleration measurement error.
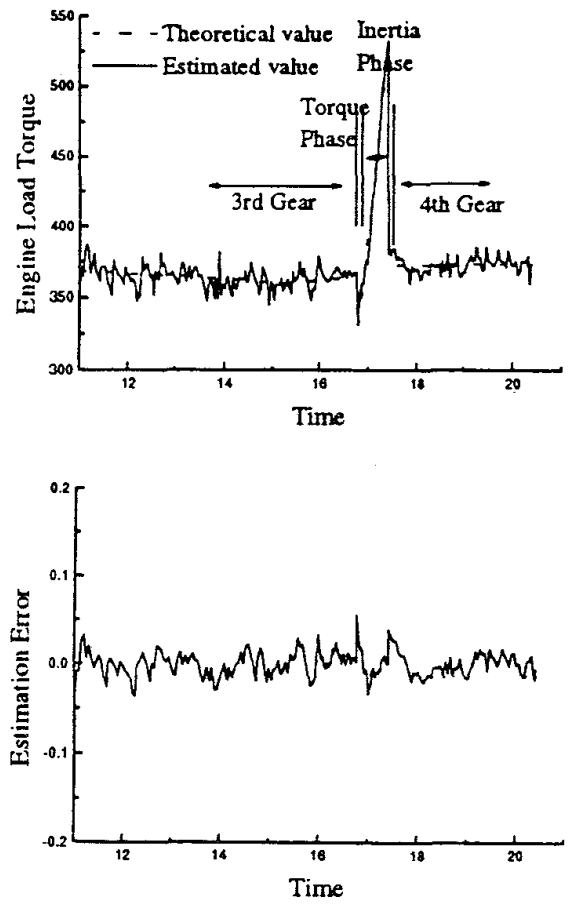

Figure 6 - The Engine Load Torque Estimation

The computer simulations show that the engine load torque estimation errors are generally slightly larger than the direct drive shaft torque estimation errors. The additional torque estimation errors are mainly caused by the measurement error of turbine acceleration. The engine load torque estimation error could be reduced by improving the accuracy of the turbine acceleration measurement. With more accurate acceleration measurement, the engine load torque estimation could be more closer to the actual values.

\section{Acknowledgments}

The authors wish to thank Ford Motor Company for sponsorship of this research, particularly Dr. Wayne J. Johnson and his staff at the Scientific Research Lab.

\section{References}

1. Pan, C.-H., \& Moskwa, J. J., 1995, "Dynamic Modeling and Simulation of the Ford AOD-E Automobile Transmission", SAE Tech. Paper 950899. 2. Young, K. D., \& Ozguner, U., 1993, "Frequency Shaping Compensator Design for Sliding Mode," Int. Journal of Control, Vol. 57, No. 5, 1005-1020. 3. Misawa, E. A., 1988, "Nonlinear State Estimation Using Sliding Observers," PhD. Dissertation, MT. 4. Misawa, E. A., \& Hedrick, J. K., "Nonlinear Observers -- A State-of-the-Art Survey," Journal of Dynamic Systems, Measurement, and Control, September, Vol. 113, pp. $\$ 344-352$.

5. Dorling, C. M., \& Zinober, A. S. I., 1986, "Two Approaches to Hyperplane Design in Multivariable Variable Structure Control Systems," International Journal of Control, Vol. 44, No. 1, 65-82.

6. Hunt, L. R., Su, R., \& Meyer, G., 1983, Design for Multi-Input Nonlinear System. Differential Geometric Cntrl. Theory, edited by R. W. Wrockett, R. S. Millman \& H. J. Sussmann (Boston: Birkhauser), 268-298. 7. Krener, A., \& Respondek, W., 1985, "Nonlinear Observers with Linearizable Error Dynamics," Soc. for Industrial and Applied Mathematics Journal of Control and Optimization, March, Vol. 23, No. 2, 197-216. 8. Walcott, B. L., Corless, M. L., \& Zak, S. H., 1987, "Comparative Study of Non-Linear State-Observation Techniques," International Journal of Control, Vol. 45, No. 6, pp. 2109-2132.

9. Woodham, C. A., \& Zinober, A. S. I., 1993, "Eigenvalue Placement in a Specified Sector for Variable Structure Control Systems," International Journal of Control, Vol. 57, No. 5, 1021-1037. 10. Zanasi, R., 1993, "Sliding Mode Using Discountinuous Control Algorithms of Integral Type," Int. Journal of Control, Vol. 57, No. 5, 1079-1099.

11. Hashimoto, H., Maruyama, K., \& Harashima, F., 1987, "A Microprocessor-Based Robot Manipulator Control with Sliding Mode," IEEE Trans. on Industrial Electronics, Vol. IE-34, No. 1. February, pp.11-18. 12. Kaidantzis, P., Rasmussen, P., Jensen, M., Vesterholm, T., \& Hendricks, E., 1993, "Advanced Nonlinear Observer Control of SI Engines," SAE Paper No. 930768

13. Slotine, J.-J. E., \& Li, W., 1991, Applied Nonlinear Control, Prentice-Hall.

14. Pan, C.H., "Powertrain Modeling and Engine Torque Estimation Using Nonlinear Observers," Ph.D. Thesis, Dept. of Mech. Engr., Univ. of Wisconsin, June 1995. 15. Demmler, A., \& Jost, K., 1992, "1993 New Vehicle Tech Highlights," Automotive Engr., Oct., pp. 59-88. 16. Pan, C.-H., Shiao, Y., \& Moskwa, J. J., 1993, "Advanced Dynamic Spark Ignition Engine Modeling for Diagnostics and Control," International Journal of Vehicle Design, Vol. 15, No. 6, 1994. 